\title{
Characterization of genome-wide H3K27ac profiles reveals a distinct $\mathrm{PM}_{2.5}$-associated histone modification signature
}

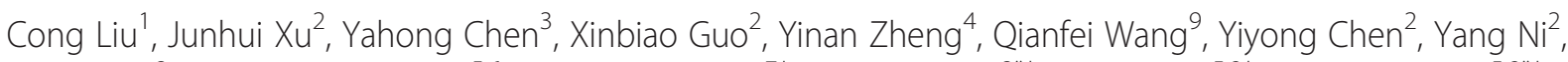
Yidan Zhu' ${ }^{2}$, Brian Thomas Joyce ${ }^{5,6}$, Andrea Baccarelli ${ }^{7 \dagger}$, Furong Deng ${ }^{2^{*}+}$, Wei Zhang ${ }^{5,8+}$ and Lifang Hou H, $^{*+}$

\begin{abstract}
Background: Current studies of environmental health suggest a link between air pollution components, such as particulate matter (PM), and various diseases. However, the specific genes and regulatory mechanisms implicated in PM-induced diseases remain largely unknown. Epigenetic systems such as covalent modification of histones in chromatin may mediate environmental factors in gene regulation. Investigating the relationships between PM exposure and histone modification status may help understand the mechanisms underlying environment-associated health conditions.

Methods: In this study, we obtained genome-wide profiles of H3K27ac (histone 3 lysine 27 acetylation), known to be an active gene regulatory histone modification marker, in blood samples collected from four Chinese individuals exposed to high or low $\mathrm{PM}_{2.5}$ (particles with diameters up to $2.5 \mu \mathrm{m}$ ).

Results: The genome-wide chromatin immunoprecipitation sequencing (ChIP-Seq) data indicated a comprehensive differential H3K27ac landscape across the individual genomes, which was associated with high $\mathrm{PM}_{2.5}$. Moreover, a substantial number of these $\mathrm{PM}_{2.5}$-associated differential H3K27ac markers were in genes involved in immune cell activation, potentially linking these epigenetic changes with air pollution-induced immune and inflammatory responses.

Conclusions: Our study provides the first genome-wide characterization of H3K27ac profiles in individuals subjected to different exposure levels of $\mathrm{PM}_{2.5}$. Future systematic investigations of the relationships between air pollutants and histone modifications in large population samples are warranted to elucidate the contributions of histone modifications to environment-associated diseases.
\end{abstract}

Keywords: Histone modification, H3K27ac, Particulate matter, Epigenetics, Environmental health, Gene regulation

\section{Background}

Air pollutants have been demonstrated to exert significant adverse health effects in populations around the world. Particulate matter (PM), which represents a mixture of solid particles and liquid droplets found in the air, in particular has been associated with increased morbidity and mortality from various diseases [1-5]. Gene dysregulation plays a fundamental role in disease pathogenesis

\footnotetext{
* Correspondence: lotus321321@126.com; I-hou@northwestern.edu ${ }^{\dagger}$ Equal contributors

${ }^{2}$ Department of Occupational and Environmental Health Sciences, School of Public Health, Peking University, Beijing 100191, China

${ }^{5}$ Department of Preventive Medicine, Northwestern University Feinberg

School of Medicine, Chicago, IL, USA

Full list of author information is available at the end of the article
}

and development. Thus investigating gene dysregulation mechanisms related to PM exposure may enhance our knowledge of air pollution-related health conditions, providing important information for disease prevention, diagnosis and treatment.

Covalent histone modifications, such as methylation and acetylation of certain amino acid residues in chromatin histones, have been shown to play an essential role in gene regulatory function by modulating chromatin structures. For example, the Encyclopedia of DNA Elements (ENCODE) Project [6] has systematically identified histone modification markers with distinct gene regulatory roles in the human genome (e.g., activation by H3K4me3 - histone

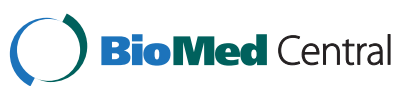

(c) 2015 Liu et al. Open Access This article is distributed under the terms of the Creative Commons Attribution 4.0 International License (http://creativecommons.org/licenses/by/4.0), which permits unrestricted use, distribution, and reproduction in any medium, provided you give appropriate credit to the original author(s) and the source, provide a link to the Creative Commons license, and indicate if changes were made. The Creative Commons Public Domain Dedication waiver (http://creativecommons.org/publicdomain/zero/1.0/) applies to the data made available in this article, unless otherwise stated. 
3 lysine 4 tri-methylation; repression by H3K9me3 - histone 3 lysine 9 tri-methylation) [7].

Furthermore, epigenetic changes including histone modifications are increasingly being linked with gene dysregulation and cellular responses induced by air pollutants, including ambient PM [8]. PM exposure was found to promote the release of inflammatory cytokines, which is further enhanced by co-treatment with a histone deacetylase inhibitor [9], indicating that differential histone acetylation could be involved in PM-mediated pro-inflammatory responses. Some reports also indicated that PM-containing environmental contaminants (e.g., nickel, chromium) contribute to dysregulated histone acetylation [10, 11]. Since there are different types of histone modifications, the ultimate effects of histone modifications related to air pollutant exposure remain unclear, and likely depend upon the exact composition of the PM. In addition, genome-wide histone modification patterns induced by air pollution has yet to be characterized. Therefore, in this study, our objective was to characterize the modification patterns of H3K27ac (histone 3 lysine 27 acetylation) associated with $\mathrm{PM}_{2.5}$ (particles with diameters up to $2.5 \mu \mathrm{m}$ ). H3K27ac has been identified as an active regulatory histone modification marker with a putative role in separating active enhancers from their poised counterparts [12]. Specifically, we used the unbiased, genome-wide Chromatin Immunoprecipitation Sequencing (ChIP-Seq) to profile H3K27ac markers across the genomes of individuals with varying $\mathrm{PM}_{2.5}$ exposure levels. Individual histone modification profiles were compared between the exposure groups to provide an overall landscape of differential H3K27ac markers associated with high $\mathrm{PM}_{2.5}$ exposure. Genes that may be regulated by these $\mathrm{PM}_{2.5}$-associated histone markers were then evaluated for their potential functions and impacts on human complex diseases/traits, by taking advantage of publicly available functional annotation databases and genome-wide association study (GWAS) results.

\section{Methods}

Study subjects, sample preparation, and ChIP-Seq assay Four healthy subjects were assigned into low or high exposure groups according to measurements of outdoor $\mathrm{PM}_{2.5}$ levels (Table 1). All study participants are Han Chinese who worked and lived in the Beijing metropolitan area. This study was approved and exempted by the Institutional

Table 1 Study subjects

\begin{tabular}{llll}
\hline Subject ID & $\begin{array}{l}\text { Exposure } \\
\text { group }\end{array}$ & $\begin{array}{l}\text { Outdoor } P M_{2.5} \\
\left(\mu \mathrm{g} / \mathrm{m}^{3}\right)\end{array}$ & $\begin{array}{l}\text { Indoor } P M_{2.5} \\
\left(\mu \mathrm{g} / \mathrm{m}^{3}\right)\end{array}$ \\
\hline 1 & low & 7 & 15 \\
2 & low & 9 & 17 \\
3 & high & 22 & 105 \\
4 & high & 52 & 131 \\
\hline
\end{tabular}

Review Board of each collaborating institution with written informed consent obtained from all subjects.

Nuclei from polymorphonuclear leukocytes of subjects were extracted using PolymorphPrep ${ }^{\mathrm{Tm}}$ (Axis-Shield, Dundee, UK). These extracted nuclei were then lysed and sonicated to produce sheared chromatin 200-600 bp long. The quality of the sheared chromatin, and sonication efficiency, were checked according to standard molecular biology protocols. The ChIP-Seq assay was then used to profile the modification levels of H3K27ac in each individual's genomes. Briefly, the final soluble chromatin was prebound with an H3K27ac antibody (Abcam, Cambridge, UK; \#ab4729). Whole-cell extract (WCE) samples untreated with the antibody (i.e., the input samples) were retained as controls. The immunoprecipitated chromatin was washed, purified and eluted. The crosslinks were then reversed. The purified DNA were prepared for sequencing using the ChIP-Seq kit according to the manufacturer's protocol (Illumina, Inc., San Diego, CA). Sequencing was performed using the Illumine HiSeq2000 platform (Illumina, Inc., San Diego, CA). The raw ChIP-Seq data have been deposited into the NCBI Sequence Read Archive (Accession Number: SRP057970).

\section{ChIP-Seq data processing and characterization}

Histone modification peaks were identified from the raw ChIP-Seq data using the next-generation sequencing analysis tools provided in the Galaxy Project [13]. Low quality reads as more than $10 \%$ of bases with quality scores less than 20 were filtered. The cleaned $101 \mathrm{bp}$ single-end sequencing reads were mapped to the human genome reference (hg19) using Bowtie2 [14]. Only nonredundant and uniquely mapped reads were retained to correct for sequencing bias. To define the H3K27ac enriched genomic regions (peaks), the model-based algorithm MACS [15] was used to compare the ChIP-Seq signal to its corresponding input sample. Peaks with overlaps in different individuals were merged into a broad peak domain using BEDTools [16]. Differential H3K27ac loci were defined as broad peaks detected in one group that were missing in another group.

To characterize the general distribution of H3K27ac profiles in these individuals, aggregate $\mathrm{H} 3 \mathrm{~K} 27 \mathrm{ac}$ profiles were generated using an in-house script. The human RefSeq [17] transcription start site (TSS) annotations and the ENCODE ChromHMM-detected enhancers derived for the lymphoblastoid cell line GM12878 [18] were downloaded from the UCSC Genome Browser (https://genome.ucsc.edu/). A window of $+/-10 \mathrm{~kb}$ from each TSS or center of each enhancer was split into 400 bins $(50 \mathrm{bp} / \mathrm{bin})$. For each bin, the normalized reads density was calculated as a log ratio of the average reads density for the ChIP sample to its corresponding input 
sample, thus generating a genome-wide H3K27ac intensity profile for either promoters or enhancers.

\section{Functional annotation analyses}

Functions of differential H3K27ac loci were predicted by analyzing the annotations of nearby genes using the Genomic Regions Enrichment of Annotations Tool (GREAT) [19]. In particular, each gene was assigned a basal regulatory domain from $5 \mathrm{~kb}$ upstream to $1 \mathrm{~kb}$ downstream of the TSS. The gene regulatory domain was extended in both directions to the next nearest gene's basal domain but no more than $100 \mathrm{~kb}$ in one direction. Each differential H3K27ac locus was associated with all genes whose regulatory domain it overlapped. Significantly enriched Gene Ontology (GO) [20] biological processes and PANTHER pathways [21] were identified under $5 \%$ false discovery rate (FDR) using a hypergeometric test. To evaluate $\mathrm{PM}_{2.5}$-associated epigenetic effects on human complex diseases/traits, differential H3K27ac loci were overlapped with trait-associated genetic variants, i.e., single nucleotide polymorphisms (SNPs) from the NHGRI GWAS Catalogue [22], which contains over 1700 curated publications of more than 12000 SNPs (Catalog Data: February 20, 2015). Significant GWAS SNP-trait associations were limited to those with nominal p-values smaller than $1.0 \times 10^{-5}$, as reported by the GWAS Catalogue.

\section{Results}

Figure 1 shows the general workflow of this study. Briefly, four individuals subjected to different $\mathrm{PM}_{2.5}$ exposure levels were profiled for genome-wide H3K27ac profiles using ChIP-Seq. A standard ChIP-Seq data analysis pipeline was performed to identify differential histone modification loci between the two exposure groups.

For each individual, both ChIP and input samples were sequenced. After conventional quality control, around 2-9 million unique reads were mapped to the reference genome (hg19) in the ChIP samples, in contrast to the 4-12 million reads in the input samples (Additional file 1: Table S1). In total, $7000 \sim 54000$ peaks were called using a stringent peak detection threshold $\mathrm{p}$-value of $p<10^{-5}$ (Additional file 2: Table S2). Among differentially modified H3K27ac loci, 1080 loci were induced in the group with high $\mathrm{PM}_{2.5}$ exposure, and 158 loci were suppressed (Additional file 3: Table S3). In general, individuals with higher $\mathrm{PM}_{2.5}$ tended to have a higher number of peaks. In addition, a similar global pattern was observed from aggregation plots of H3K27ac on promoter and enhancer regions (Fig. 2). H3K27ac peaks were clearly overlapped in promoter and enhancer regions, consistent with the putative role of H3K27ac as a promoter and enhancer marker. Both the TSS and enhancer peaks were higher in the individuals with high $\mathrm{PM}_{2.5}$ exposure compared to low-exposed individuals. These findings

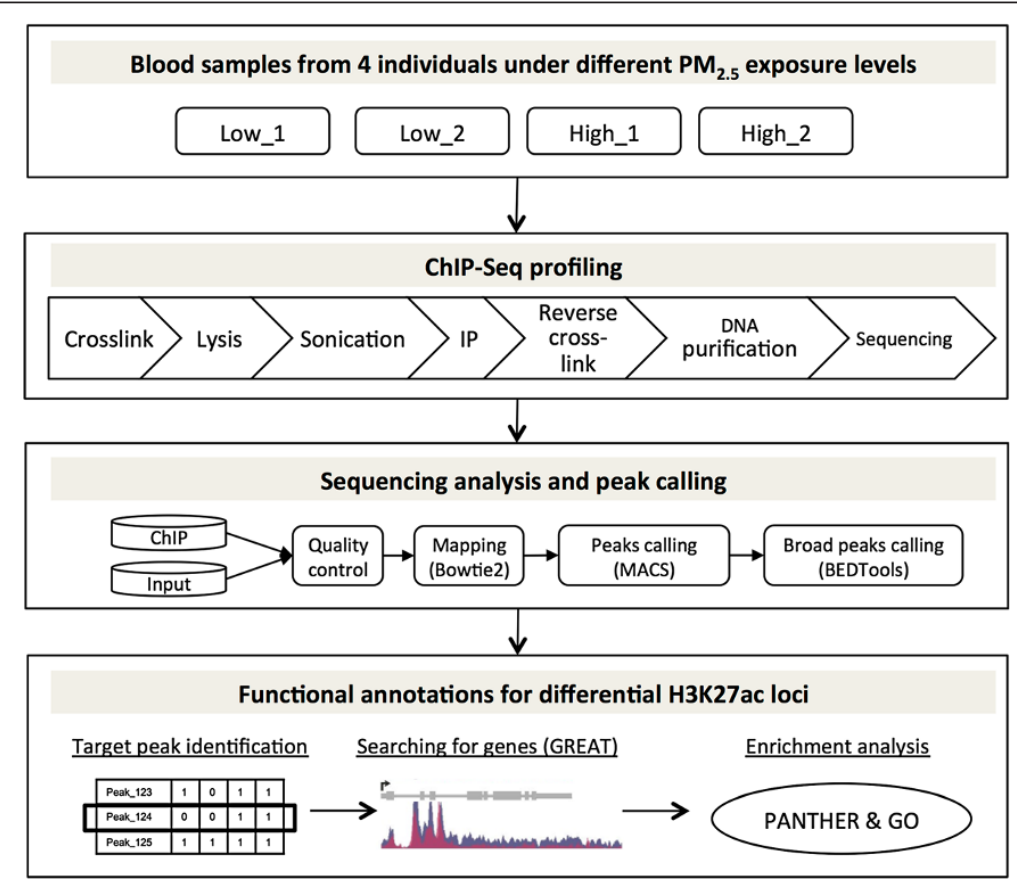

Fig. 1 Overview of the study design and analysis workflow. Nuclei were extracted from blood samples of four individuals under low or high exposure

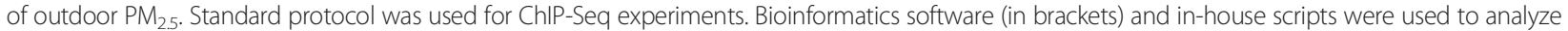
the sequencing data. The functions of differential H3K27ac loci were evaluated using public databases. ChIP: chromatin immunoprecipitation; IP: immunoprecipitation; GO: gene ontology 

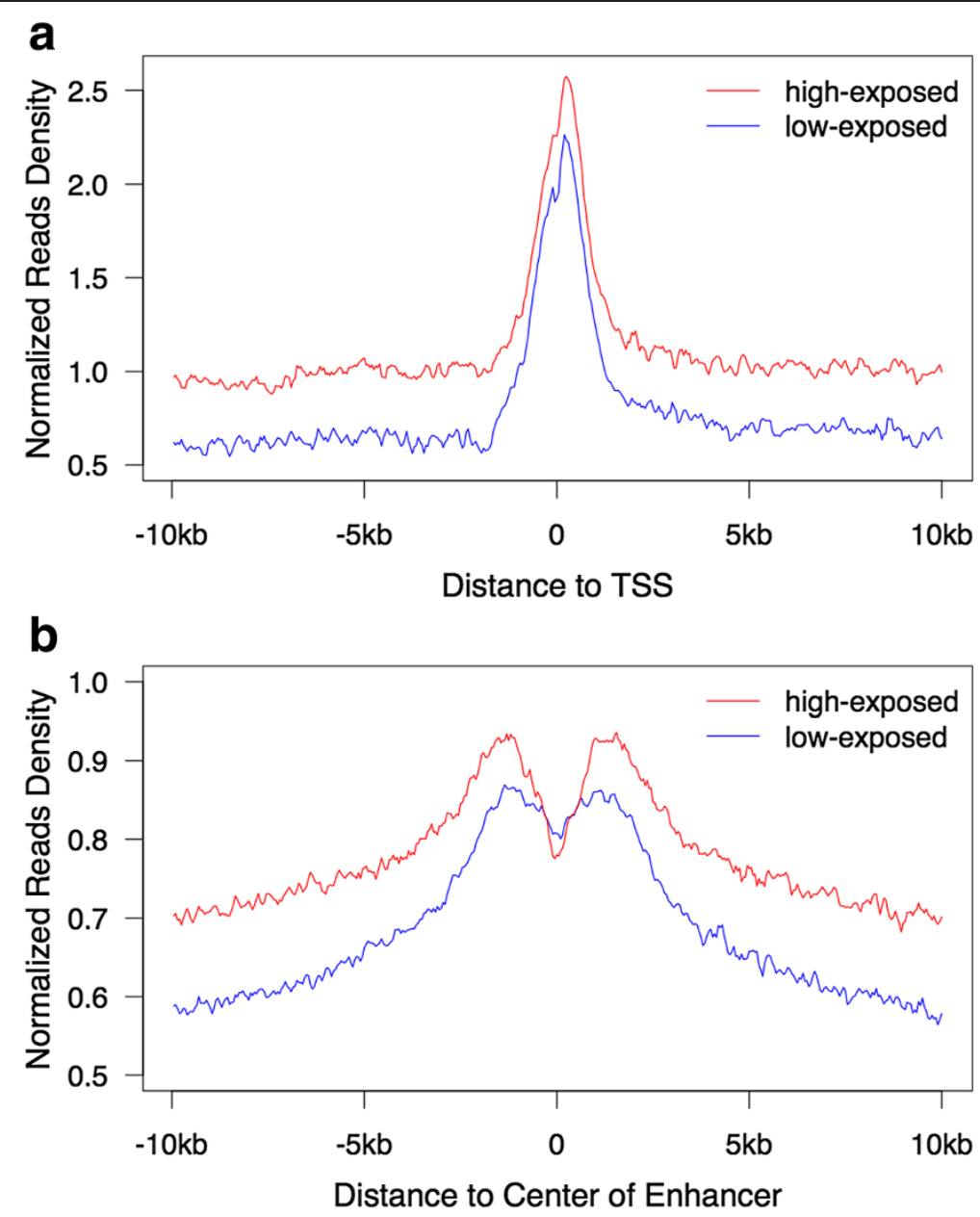

Fig. 2 Global profiles of H3K27ac overlapped with promoters and enhancers. Global H3K27ac signal densities normalized by input were determined in a $20 \mathrm{~kb}$-window surrounding a the center of ENCODE-detected enhancers; and $\mathbf{b}$ the TSS of human RefSeq genes. The red curve represents the average H3K27ac signal density in the high-exposed group and the blue curve represents the average density in the low-exposed group. Both promoters and enhancers show global elevated H3K27ac modification levels in the individuals exposed to higher PM 2.5 (red). TSS: transcription start site

could indicate global enhancement of gene expression due to the exposure to $\mathrm{PM}_{2.5}$ pollutants.

Gene set enrichment analysis was performed to evaluate the genes annotated by the identified differential H3K27ac loci using the GREAT tools. Additional file 4: Table S4 shows significantly enriched GO biological processes and PANTHER pathways. We found that most of the associated genes were involved in the activation of cellular responses to wounding and stimulus, suggesting an enhancer-mediated cell activation mechanism in response to higher $\mathrm{PM}_{2.5}$ exposure. We also found differential H3K27ac loci were most significantly enriched in pathways related to immune response, including T-cell and B-cell activation (Fig. 3). Interestingly, we found that a pathway related to Alzheimer's disease was enriched in our results.

A previous study showed that complex trait-associated variants were enriched in specific histone marks [23].
We also found 11 complex trait-associated genetic variants overlapping with our identified $\mathrm{PM}_{2.5}$-associated epigenetic signature (Table 2). Diseases such as Alzheimer's disease and inflammatory bowel disease (IBD) were found to have overlapping GWAS-identified loci with our $\mathrm{PM}_{2.5}$-associated H3K27ac markers.

\section{Discussion}

Accumulating evidence has demonstrated that PM is able to induce systemic inflammatory responses by altering the expression of specific genes [24-28]. For example, Ovrevik, et al. [26] found that particulate air pollution up-regulated inflammation-related chemokines and cytokines in a bronchial epithelial cell line. In a paired sampling study design, Wang, et al. [25] showed that levels of systemic inflammatory responses were significantly increased in the peripheral blood of a population exposed to $\mathrm{PM}_{2.5}$. Our observation of $\mathrm{PM}_{2.5}$-associated epigenetic change is consistent with 


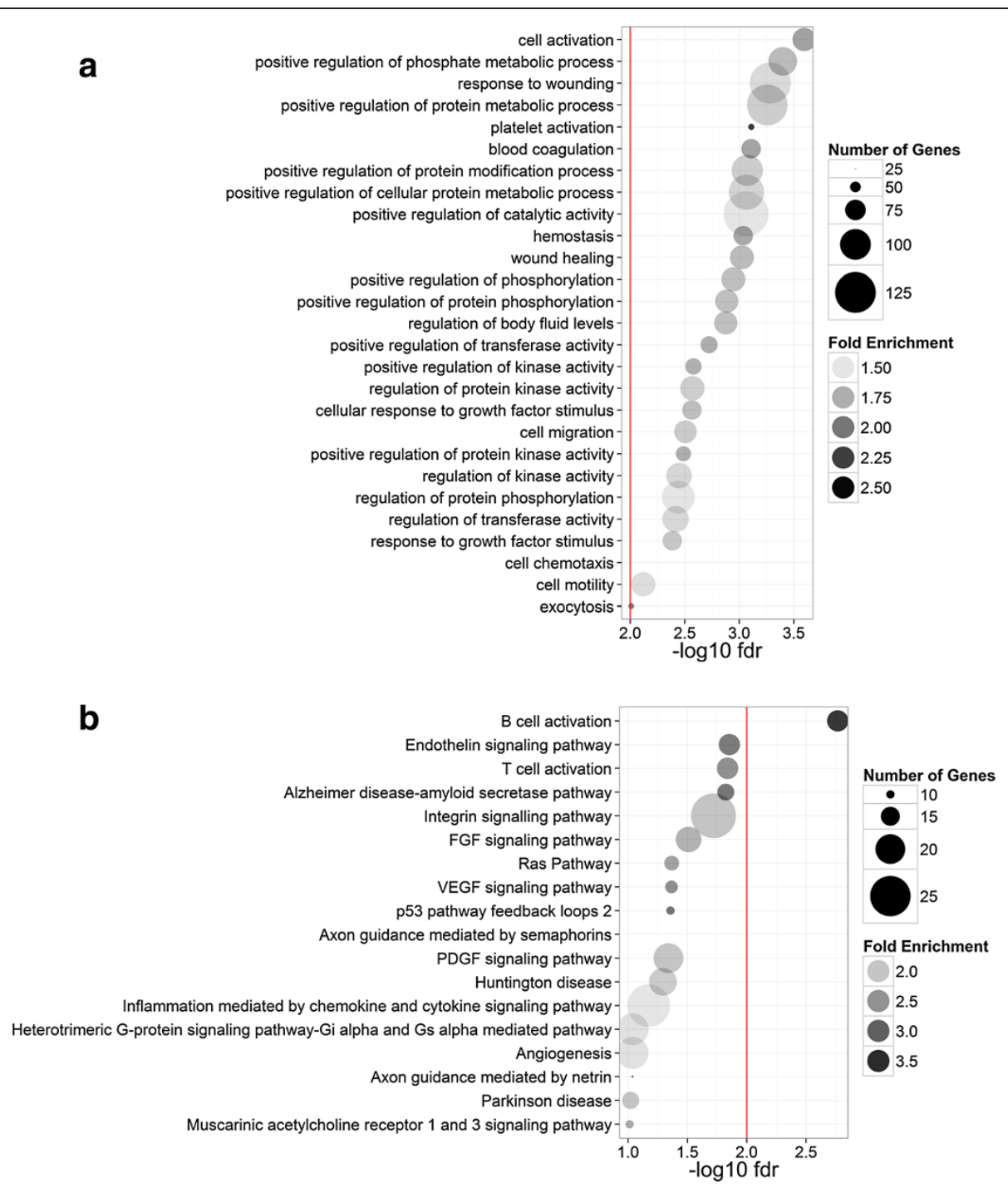

Fig. 3 Enriched functional annotations among differential H3K27ac loci. Proximal gene enrichments for differential H3K27ac loci were analyzed using a GO biological processes; and $\mathbf{b}$ PANTHER pathways. Circle size is proportional to the number of identified genes. Circle transparency is proportional to fold enrichment relative to the human genome. Functional annotations were ordered by FDR derived from hypergeometric test. Red vertical lines indicate the cutoff at $5 \%$ FDR. GO: gene ontology; FDR: false discovery rate

previous studies. Specifically, various biological processes related to inflammatory or immune cell activation and inflammation pathways such as CXC-chemokine receptors appeared to be activated via altered acetylation levels of H3K27 occurring after exposure to $\mathrm{PM}_{2.5}$.

$\mathrm{PM}_{2.5}$ is also notoriously implicated in blood coagulation. Previous studies $[29,30]$ demonstrated that inhalation of some PM components may be responsible for altering red cell adhesiveness, inducing endothelial dysfunction, and increasing blood coagulation, all of which offers biological mechanisms for the observed cardiovascular effects of particulate air pollution exposure. Our study found that increased exposures to $\mathrm{PM}_{2.5}$ might cause increased acetylation levels of H3K27ac markers for specific genes involved in platelet activation, blood coagulation and hemostasis. Certain pathways are likely to be activated via epigenetic regulation, thus leading to increased coagulation of red cells, leading to various cardiovascular diseases in turn.

Air pollution has been considered a risk factor for both complex neurodegenerative diseases such as Alzheimer's disease and Parkinson's disease, and monogenic neurological disorders such as Huntington's disease [31, 4, 32]. Our observation that there is an interaction between $\mathrm{PM}_{2.5}$ and epigenetic regulation offers a possible explanation for these nervous system diseases. Certain differential H3K27ac loci are potential regulators for genes involved in the Alzheimer's disease-amyloid secretase pathway, Huntington disease and Parkinson disease. Two Alzheimer's disease-associated genetic 
Table 2 Overlap of complex trait-associated genetic variants and the identified PM 2.5 -asociated H3K27ac loci

\begin{tabular}{|c|c|c|c|c|c|}
\hline Complex trait & SNP ID & Region & Reported gene & Context & $\begin{array}{l}\text { PubMed } \\
\text { reference }\end{array}$ \\
\hline Inflammatory bowel disease & rs12654812 & $5 q 35.3$ & DOK3 & intron & 23128233 \\
\hline Renal function-related traits & rs12654812 & $5 q 35.3$ & RGS14 & intron & 22797727 \\
\hline $\begin{array}{l}\text { Suicide attempts in depression } \\
\text { or bipolar disorder }\end{array}$ & rs17173608 & $7 q 36.1$ & RARRES2 & intron & 24964207 \\
\hline IgG glycosylation & rs11994937 & $8 q 12.1$ & - & intron & 23382691 \\
\hline Fibrinogen & rs7464572 & $8 q 24.3$ & PLEC1 & intron & 23969696 \\
\hline Metabolic traits & rs6558295 & $8 q 24.3$ & OPLAH & intron & 21886157 \\
\hline $\begin{array}{l}\text { Adverse response to chemotherapy } \\
\text { (neutropenia/leucopenia) } \\
\text { (paclitaxel + carboplatin) }\end{array}$ & rs10785877 & $9 q 34.2$ & RXRA & - & 23648065 \\
\hline \multirow[t]{2}{*}{ Alzheimer's disease } & rs3764650 & 19p13.3 & $\mathrm{ABCA7}$ & intron & 21460840 \\
\hline & rs115550680 & 19p13.3 & ABCA7, HMHA1, GRIN3B & intron & 23571587 \\
\hline Red blood cell traits & rs737092 & $20 q 13.31$ & RBM38 & - & 23222517 \\
\hline Cognitive decline (age-related) & rs9980664 & $21 \mathrm{q} 22.11$ & Intergenic & - & 24468470 \\
\hline
\end{tabular}

variants (rs3764650, rs115550680) [33, 34] were found to fall into the differential H3K27ac loci in our study. Another variant (rs17173608) associated with depression and bipolar disorder [35] was also found to overlap with a $\mathrm{PM}_{2.5}$-associated enhancer region. Our results suggest that $\mathrm{PM}_{2.5}$ may play an important role in deregulating nervous system functions via its ability to alter the acetylation levels of related enhancers.

By linking differentially modified H3K27ac loci to complex-trait loci identified by GWAS studies [22], we found that $\mathrm{PM}_{2.5}$-associated epigenetic changes may help improve our understanding of human complex diseases/ traits. For example, ambient air pollution has been reported to correlate with hospitalizations for IBD [36], while our finding suggested that a previously identified inflammatory bowel disease-associated variant (rs12654812) overlapped with a $\mathrm{PM}_{2.5}$-associated $\mathrm{H} 3 \mathrm{~K} 27 \mathrm{ac}$ locus, thus air pollution-associated IBD is likely mediated through this histone modification marker.

\section{Conclusions}

Our study constitutes the first genome-wide characterization of H3K27ac profiles in individuals who are subjected to different exposure levels of $\mathrm{PM}_{2.5}$. Our findings reveal a global elevation of the enhancer-associated H3K27ac markers in individuals exposed to relatively high levels of $\mathrm{PM}_{2.5}$. Furthermore, certain immune response and inflammation-related genes are likely mediated via H3K27ac makers under $\mathrm{PM}_{2.5}$ exposure. We recognize that this study had a number of limitations. Due to the limited sample size, it may not be plausible to draw firm conclusions from these data yet. In addition, epigenetic markers may be affected by possible confounding factors, such as age, gender, and race. Statistically, potential false positives from conducting multiple tests are a concern as with any high-throughput technology. However, as the first genome-wide H3K27ac landscape in people exposed to high levels of $\mathrm{PM}_{2.5}$, our investigation suggests a trend that increasing exposure to $\mathrm{PM}_{2.5}$ may enhance global gene activity. Changes in certain H3K27ac modification loci may affect local gene expression, which in turn could lead to a variety of diseases. Large-scale studies in the future are warranted to comprehensively evaluate and validate the genes and pathways influenced by $\mathrm{PM}_{2.5}$ through H3K27ac and other histone modifications.

\section{Additional files}

Additional file 1: Table S1. Summary of sequencing and mapping results. (DOCX $20 \mathrm{~kb}$ )

Additional file 2: Table S2. Summary of peak calling results. (DOCX $14 \mathrm{~kb}$ )

Additional file 3: Table S3. Complete list of differentially modified H3K27ac loci. (XLSX 69 kb)

Additional file 4: Table S4. Complete list of functional annotations. (XLSX $16 \mathrm{~kb})$

\section{Abbreviations}

ChIP: Chromatin immunoprecipitation; ENCODE: Encyclopedia of DNA elements; GO: Gene ontology; GREAT: Genomic regions enrichment of annotations tool; GWAS: Genome-wide association study; H3K27ac: Histone 3 lysine 27 acetylation; H3K4me3: Histone 3 lysine 4 tri-methylation; H3K9me3: Histone 3 lysine 9 tri-methylation; IBD: Inflammatory bowel disease; PM: Particulate matter; $\mathrm{PM}_{2.5}$ : Particles with diameters up to $2.5 \mu \mathrm{m}$; SNP: Single nucleotide polymorphism; TSS: Transcription start site; WCE: Whole-cell extract.

\section{Competing interests}

The authors declare that they have no competing interests.

\section{Authors' contributions}

$L H, A B, F D, X G$, and $W Z$ conceived the study and its design, and coordinated sample collection. WZ, CL performed data analysis, and drafted the initial manuscript. QW, YHC, YYC, YN, and YZ provided technical input to sample preparation, data collection, and exposure assessment. JX and QW 
conducted the laboratory assays. YZ participated in data collection and analysis. BTJ contributed to manuscript preparation. All authors read and approved the final manuscript.

\section{Acknowledgements}

This work was supported, in part, by grants from the National Institutes of Health: ES00002 and R21ES020010 (to LH, AB), R21HG006367 (to WZ), and The Robert H. Lurie Comprehensive Cancer Center-Developmental Funds P30CA060553 (to WZ).

\section{Author details}

'Department of Bioengineering, University of Illinois at Chicago, Chicago, IL, USA. ${ }^{2}$ Department of Occupational and Environmental Health Sciences, School of Public Health, Peking University, Beijing 100191, China. ${ }^{3}$ Respiratory Department, Peking University Third Hospital, Beijing, China. ${ }^{4}$ Institute for Public Health and Medicine, Northwestern University Feinberg School of Medicine, Chicago, IL, USA. ${ }^{5}$ Department of Preventive Medicine, Northwestern University Feinberg School of Medicine, Chicago, IL, USA. ${ }^{6}$ Division of Epidemiology/Biostatistics, School of Public Health, University of Illinois at Chicago, Chicago, IL, USA. ${ }^{7}$ Department of Environmental Health, Harvard T.H. Chan School of Public Health, Boston, MA, USA. ${ }^{8}$ The Robert H. Lurie Comprehensive Cancer Center, Northwestern University Feinberg School of Medicine, 680 N. Lake Shore Dr., Suite 1400, Chicago, IL 60611, USA. ${ }^{9}$ Key Laboratory of Genomic and Precision Medicine, Beijing Institute of Genomics, Chinese Academy of Sciences, Beijing 100101, China.

Received: 14 May 2015 Accepted: 31 July 2015

\section{Published online: 15 August 2015}

\section{References}

1. Brook RD, Franklin B, Cascio W, Hong Y, Howard G, Lipsett M, et al. Air pollution and cardiovascular disease: a statement for healthcare professionals from the Expert Panel on Population and Prevention Science of the American Heart Association. Circulation. 2004;109(21):2655-71. doi:10.1161/01.CIR.0000128587.30041.C8.

2. Hou L, Zhang X, Wang D, Baccarelli A. Environmental chemical exposures and human epigenetics. Int J Epidemiol. 2012;41(1):79-105. doi:10.1093/ije/dyr154.

3. Pope 3rd CA, Ezzati M, Dockery DW. Fine particulate air pollution and life expectancies in the United States: the role of influential observations. J Air Waste Manage Assoc. 2013;63(2):129-32.

4. Block ML, Calderon-Garciduenas L. Air pollution: mechanisms of neuroinflammation and CNS disease. Trends Neurosci. 2009;32(9):506-16. doi:10.1016/j.tins.2009.05.009.

5. Cantone L, Nordio F, Hou L, Apostoli P, Bonzini M, Tarantini L, et al. Inhalable metal-rich air particles and histone H3K4 dimethylation and H3K9 acetylation in a cross-sectional study of steel workers. Environ Health Perspect. 2011;119(7):964-9. doi:10.1289/ehp.1002955.

6. Consortium EP. An integrated encyclopedia of DNA elements in the human genome. Nature. 2012;489(7414):57-74. doi:10.1038/nature11247.

7. Barski A, Cuddapah S, Cui K, Roh TY, Schones DE, Wang Z, et al. Highresolution profiling of histone methylations in the human genome. Cell. 2007;129(4):823-37. doi:10.1016/j.cell.2007.05.009.

8. Wang T, Garcia JG, Zhang W. Epigenetic regulation in particulate mattermediated cardiopulmonary toxicities: a systems biology perspective. Current Pharmacogenomics Personalized Med. 2012;10(4):314-21.

9. Gilmour PS, Rahman I, Donaldson K, MacNee W. Histone acetylation regulates epithelial IL-8 release mediated by oxidative stress from environmental particles. Am J Physiol Lung Cell Mol Physiol. 2003;284(3): L533-40. doi:10.1152/ajplung.00277.2002.

10. Ke Q, Davidson T, Chen H, Kluz T, Costa M. Alterations of histone modifications and transgene silencing by nickel chloride. Carcinogenesis. 2006;27(7):1481-8. doi:10.1093/carcin/bgl004.

11. Schnekenburger M, Talaska G, Puga A. Chromium cross-links histone deacetylase 1-DNA methyltransferase 1 complexes to chromatin, inhibiting histone-remodeling marks critical for transcriptional activation. Mol Cell Biol. 2007;27(20):7089-101. doi:10.1128/MCB.00838-07.

12. Creyghton MP, Cheng AW, Welstead GG, Kooistra T, Carey BW, Steine EJ, et al. Histone H3K27ac separates active from poised enhancers and predicts developmental state. Proc Natl Acad Sci U S A. 2010;107(50):21931-6. doi:10.1073/pnas.1016071107

13. Goecks J, Nekrutenko A, Taylor J, Galaxy T. Galaxy: a comprehensive approach for supporting accessible, reproducible, and transparent computational research in the life sciences. Genome Biol. 2010;11(8):R86. doi:10.1186/gb-2010-11-8-r86.

14. Langmead B, Salzberg SL. Fast gapped-read alignment with Bowtie 2. Nat Methods. 2012;9(4):357-9. doi:10.1038/nmeth.1923.

15. Feng J, Liu T, Qin B, Zhang Y, Liu XS. Identifying ChIP-seq enrichment using MACS. Nat Protoc. 2012;7(9):1728-40. doi:10.1038/nprot.2012.101.

16. Quinlan AR, Hall IM. BEDTools: a flexible suite of utilities for comparing genomic features. Bioinformatics. 2010;26(6):841-2. doi:10.1093/bioinformatics/btq033.

17. Pruitt KD, Tatusova T, Maglott DR. NCBI Reference Sequence (RefSeq): a curated non-redundant sequence database of genomes, transcripts and proteins. Nucleic Acids Res. 2005;33(Database issue):D501-4. doi:10.1093/nar/gki025

18. Ernst J, Kheradpour P, Mikkelsen TS, Shoresh N, Ward LD, Epstein CB, et al. Mapping and analysis of chromatin state dynamics in nine human cell types. Nature. 2011;473(7345):43-9. doi:10.1038/nature09906.

19. McLean CY, Bristor D, Hiller M, Clarke SL, Schaar BT, Lowe CB, et al. GREAT improves functional interpretation of cis-regulatory regions. Nat Biotechnol. 2010;28(5):495-501. doi:10.1038/nbt.1630.

20. Gene OC. Gene ontology consortium: going forward. Nucleic Acids Res. 2015:43(Database issue):D1049-56. doi:10.1093/nar/gku1179.

21. Mi H, Muruganujan A, Thomas PD. PANTHER in 2013: modeling the evolution of gene function, and other gene attributes, in the context of phylogenetic trees. Nucleic Acids Res. 2013;41(Database issue):D377-86. doi:10.1093/nar/gks1118.

22. Hindorff LA, Sethupathy P, Junkins HA, Ramos EM, Mehta JP, Collins FS, et al. Potential etiologic and functional implications of genome-wide association loci for human diseases and traits. Proc Natl Acad Sci U S A. 2009;106(23):9362-7. doi:10.1073/pnas.0903103106.

23. Trynka G, Sandor C, Han B, Xu H, Stranger BE, Liu XS, et al. Chromatin marks identify critical cell types for fine mapping complex trait variants. Nat Genet. 2013:45(2):124-30. doi:10.1038/ng.2504.

24. Tao F, Gonzalez-Flecha B, Kobzik L. Reactive oxygen species in pulmonary inflammation by ambient particulates. Free Radic Biol Med. 2003;35(4):327-40.

25. Wang Z, Neuburg D, Li C, Su L, Kim JY, Chen JC, et al. Global gene expression profiling in whole-blood samples from individuals exposed to metal fumes. Environ Health Perspect. 2005;113(2):233-41.

26. Ovrevik J, Lag M, Holme JA, Schwarze PE, Refsnes M. Cytokine and chemokine expression patterns in lung epithelial cells exposed to components characteristic of particulate air pollution. Toxicology. 2009;259 (1-2):46-53. doi:10.1016/j.tox.2009.01.028.

27. Shoenfelt J, Mitkus RJ, Zeisler R, Spatz RO, Powell J, Fenton MJ, et al. Involvement of TLR2 and TLR4 in inflammatory immune responses induced by fine and coarse ambient air particulate matter. J Leukoc Biol. 2009;86 (2):303-12. doi:10.1189/jlb.1008587.

28. Tchou-Wong KM, Kiok K, Tang Z, Kluz T, Arita A, Smith PR, et al. Effects of nickel treatment on H3K4 trimethylation and gene expression. PLoS One. 2011;6(3), e17728. doi:10.1371/journal.pone.0017728.

29. Seaton A, Soutar A, Crawford V, Elton R, McNerlan S, Cherrie J, et al. Particulate air pollution and the blood. Thorax. 1999;54(11):1027-32.

30. Ruckerl R, Ibald-Mulli A, Koenig W, Schneider A, Woelke G, Cyrys J, et al. Air pollution and markers of inflammation and coagulation in patients with coronary heart disease. Am J Respir Crit Care Med. 2006;173(4):432-41. doi:10.1164/rccm.200507-11230C.

31. Migliore L, Coppede F. Environmental-induced oxidative stress in neurodegenerative disorders and aging. Mutat Res. 2009;674(1-2):73-84. doi:10.1016/j.mrgentox.2008.09.013.

32. Finkelstein MM, Jerrett M. A study of the relationships between Parkinson's disease and markers of traffic-derived and environmental manganese air pollution in two Canadian cities. Environ Res. 2007;104(3):420-32. doi:10.1016/j.envres.2007.03.002.

33. Hollingworth $P$, Harold D, Sims R, Gerrish A, Lambert JC, Carrasquillo MM, et al. Common variants at ABCA7, MS4A6A/MS4A4E, EPHA1, CD33 and CD2AP are associated with Alzheimer's disease. Nat Genet. 2011;43(5):429-35. doi:10.1038/ng.803.

34. Reitz C, Jun G, Naj A, Rajbhandary R, Vardarajan BN, Wang LS, et al. Variants in the ATP-binding cassette transporter (ABCA7), apolipoprotein E 4, and 
the risk of late-onset Alzheimer disease in African Americans. Jama. 2013;309(14):1483-92. doi:10.1001/jama.2013.2973.

35. Mullins N, Perroud N, Uher R, Butler AW, Cohen-Woods S, Rivera M, et al. Genetic relationships between suicide attempts, suicidal ideation and major psychiatric disorders: a genome-wide association and polygenic scoring study. Am J Med Genet B Neuropsychiatr Genet. 2014;165B(5):428-37. doi:10.1002/ajmg.b.32247.

36. Ananthakrishnan AN, McGinley EL, Binion DG, Saeian K. Ambient air pollution correlates with hospitalizations for inflammatory bowel disease: an ecologic analysis. Inflamm Bowel Dis. 2011;17(5):1138-45. doi:10.1002/ibd.21455.

\section{Submit your next manuscript to BioMed Central} and take full advantage of:

- Convenient online submission

- Thorough peer review

- No space constraints or color figure charges

- Immediate publication on acceptance

- Inclusion in PubMed, CAS, Scopus and Google Scholar

- Research which is freely available for redistribution 